\title{
Recombination between Photogenerated and Electrode-Induced Charges Dominates the Fill Factor Losses in Optimized Organic Solar Cells
}

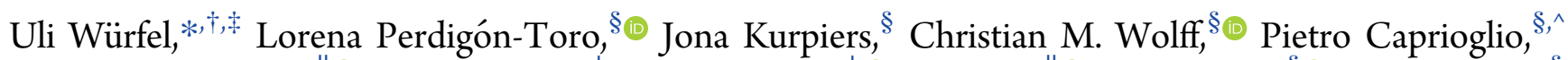

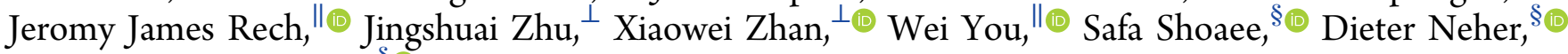 \\ and Martin Stolterfoht ${ }^{*}, \S 0$ \\ ${ }^{\dagger}$ Fraunhofer Institute for Solar Energy Systems ISE, Heidenhofstr. 2, 79110 Freiburg, Germany \\ ${ }^{\ddagger}$ Freiburg Materials Research Center FMF, Albert-Ludwigs-Universität Freiburg, Stefan-Meier-Str. 21, 79104 Freiburg, Germany \\ ${ }^{\S}$ Institut für Physik und Astronomie, Universität Potsdam, Karl-Liebknecht-Straße 24-25, 14476 Potsdam-Golm, Germany \\ "Department of Chemistry, University of North Carolina at Chapel Hill, Chapel Hill, North Carolina 27599-3290, United States \\ ${ }^{\perp}$ Department of Materials Science and Engineering, College of Engineering, Key Laboratory of Polymer Chemistry and Physics of \\ Ministry of Education, Peking University, Beijing 100871, China \\ ^Young Investigator Group Perovskite Tandem Solar Cells, Helmholtz-Zentrum Berlin, Kekuléstr. 5, 12489 Berlin, Germany
}

ABSTRACT: Charge extraction in organic solar cells (OSCs) is commonly believed to be limited by bimolecular recombination of photogenerated charges. However, the fill factor of OSCs is usually almost entirely governed by recombination processes that scale with the first order of the light intensity. This linear loss was often interpreted to be a consequence of geminate or trap-assisted recombination. Numerical simulations show that this linear dependence is a direct consequence of the large amount of excess dark charge near the contact. The first-order losses increase with decreasing mobility of minority carriers, and we discuss the impact of several material and device parameters on this loss mechanism. This work highlights that OSCs are especially vulnerable to injected charges as a result of their poor charge transport properties. This implies that dark charges need to be better accounted for when interpreting electro-optical measurements and charge collection based on simple figures of merit.

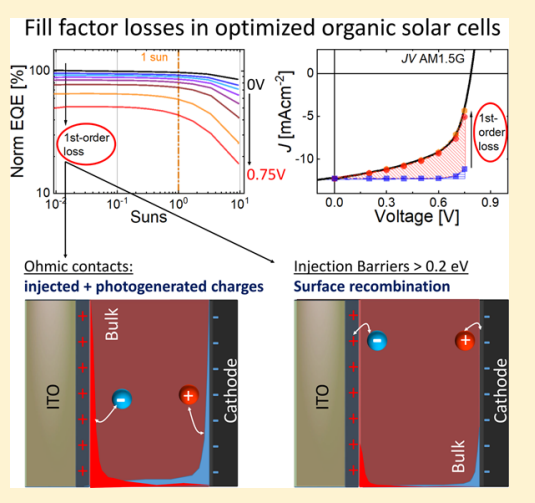

$\mathrm{O}$ rganic semiconductors are promising materials for photovoltaic energy conversion and other electronic devices. ${ }^{1,2}$ In particular with the emergence of nonfullerene acceptors, ${ }^{3,4}$ organic solar cells (OSCs) have experienced an unprecedented rise in power conversion efficiencies (PCEs) within the last 2 years, now reaching up to $15.7 \%$. ${ }^{5}$ However, the comparatively low fill factor $(<80 \%)$ of OSCs with absorber layer thicknesses relevant for the application of printing technologies still represents a major challenge for commercialization. Therefore, it is essential to understand how well charges are extracted and where they are lost in the device. ${ }^{6,7}$ Over the years, many different models have been used to explain the FF losses in OSCs, such as bimolecular recombination, trap-assisted recombination, and/or fielddependent CT-state dissociation. Recently, much evidence emerged that it is bimolecular recombination of free charge carriers that leads to fill factor losses because of the comparatively slow charge transport in the disordered organic semiconductors. ${ }^{8}$ This causes an accumulation of charge carriers and hence increases recombination losses ${ }^{6,9}$ or leads to field-free regions. ${ }^{10}$ The competition of extraction $\left(k_{\mathrm{e}}\right)$ and second-order bimolecular recombination $\left(k_{\mathrm{r}} \sim k_{2} n p\right)$ can be described with simple analytical expressions or figures of merit (FOMs); $;^{7,9,11,12}$ and these FOMs have been successfully applied to a large number of OSCs from various groups. ${ }^{7,9,11,12}$ However, intensity-dependent photocurrent (IPC) and related measurements in steady-state might suggest an entirely

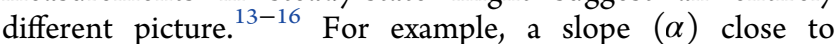
unity, fitted to the IPC plotted in log-log, is often interpreted to be a consequence of a dominant monomolecular recombination process or negligible bimolecular recombination. ${ }^{17-19}$ On the basis of this approach, several groups have proposed techniques to quantify bimolecular recombination losses from the deviation of $\alpha$ from unity. ${ }^{12,20,21}$ Similarly, early studies by Cowan et al. ${ }^{13}$ showed that the photocurrent $\left(J_{\mathrm{ph}}\right)$ losses scale mostly linearly with light intensity (I) from shortcircuit conditions to voltages beyond the maximum power point. The first-order recombination losses manifested thereby

Received: April 24, 2019

Accepted: May 31, 2019

Published: May 31, 2019 
Table 1. Photovoltaic Performance Parameters of Organic Solar Cells ${ }^{a}$

\begin{tabular}{|ccccc}
\hline Device & $J_{\mathrm{SC}}\left[\mathrm{mA} \mathrm{cm}^{-2}\right]$ & FF $[\%]$ & $\mathrm{V}_{\mathrm{OC}}[\mathrm{V}]$ & PCE $[\%]$ \\
\hline PTB7:PC & & & \\
FTA $\mathrm{BM}(1: 1.5), d=80 \mathrm{~nm}$ & $13.2 \pm 1.3(15.3)$ & $56.5 \pm 2.1(56.1)$ & $0.777 \pm 0.01(0.767)$ & $5.8 \pm 0.4(6.6)$ \\
FTAC $(1: 1.5), d=100 \mathrm{~nm}$ & $17.4 \pm 0.7(18.6)$ & $68 \pm 2.7(67.3)$ & $0.847 \pm 0.004(0.849)$ & $10 \pm 0.3(10.6)$
\end{tabular}

${ }^{a}$ Average power conversion efficiencies of the studied organic solar cells with optimized active layer thickness $(d)$ as calculated from 18 devices for each blend including the standard errors of the short-circuit current density $\left(J_{\mathrm{SC}}\right)$, open-circuit voltage $\left(V_{\mathrm{OC}}\right)$ and fill factor (FF). Record parameters are displayed in parentheses.
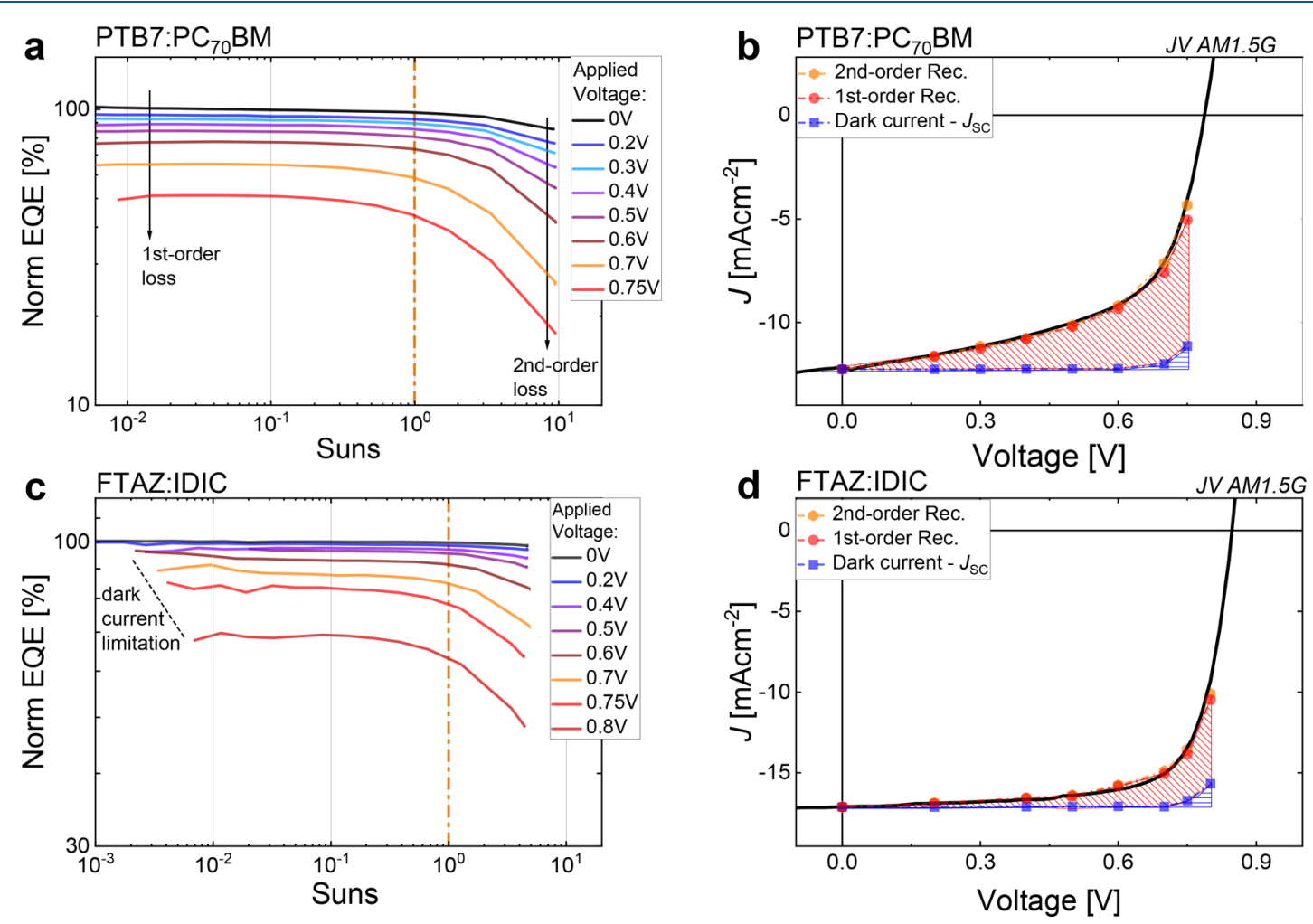

Figure 1. Intensity-dependent external quantum efficiency (EQE) at different applied voltages from short-circuit to close to open-circuit conditions for (a) $80 \mathrm{~nm}$ thick PTB7:PC $70 \mathrm{BM}(1: 1.5 \mathrm{w} / \mathrm{w})$ and (c) $100 \mathrm{~nm}$ thick FTAZ:IDIC $(1: 1.5 \mathrm{w} / \mathrm{w})$ cells. The EQE is obtained from the ratio of the photocurrent (light minus the dark current) and the illumination intensity and plotted versus equivalent suns. The 1-sun equivalent laser power (at the used excitation wavelength of $445 \mathrm{~nm}$ ) is defined where the solar cell current output matches the $J_{\mathrm{SC}}$ under AM1.5G irradiation. The corresponding JV curves of the PTB7:PC $70 \mathrm{BM}(\mathrm{b})$ and the FTAZ:IDIC (d) blends are shown on the right. The FF losses are decoupled into contributions from first- and second-order recombination processes, as well as the dark current. It is evident that the shape of the $J V$-curve is determined by first-order recombination losses resulting in intensity-independent FF losses in both systems.

as a fill factor (FF) that is almost independent of light intensity. These losses were attributed to nongeminate recombination of mobile carriers with interfacial traps, while other groups have also drawn similar conclusions. ${ }^{13,15,22-24}$ These results seem to be largely inconsistent with significant bimolecular recombination (being a second-order process with respect to the free charge carrier density) which would lead to a strong dependence of the fill factor on the light intensity. However, we note a few earlier publications pointed out that a linear dependence of recombination on the light intensity does not imply that the underlying recombination process depends linearly on the free carrier density. ${ }^{25}$ Nevertheless, other studies have attributed these first-order losses to an electricfield-dependent charge-transfer state (CT) dissociation governing the current-density versus voltage $(J V)$ characteristics. $^{26-28}$ However, this appears to be in contrast to the results of many transient studies, e.g., time-delayed-collection field (TDCF) experiments ${ }^{9,29-31}$ or ultrafast spectroscopic measurements $^{32,33}$ where an electric field-independent dissociation of CT states was often found, at least in relatively efficient bulk-heterojunction solar cells. ${ }^{4,9,34}$ Considering these inconsistencies and different explanations of the FF, a conclusive understanding about the main photocurrent losses in the power-generating regime remains an important, yet challenging task.

Recombination Order along the JV Curve. To assess the recombination order in the power-generating regime of the $J V$ curve, we employed IPC measurements (see Supplementary Methods in the Supporting Information). ${ }^{16,35}$ In the following, we will refer to the recombination order with respect to the light intensity (the terminology is further discussed in Supplementary Note 1 in the Supporting Information). First, we demonstrate the IPC results on an archetypical organic solar cell blend thieno[3,4- $b]$ thiophene/benzodithiophene: $[6,6]$-phenyl $\mathrm{C}_{71}$-butyric acid methyl ester (PTB7:PC ${ }_{70} \mathrm{BM}$ $1: 1.5),{ }^{36}$ as well as on a high-efficiency nonfullerene system FTAZ:IDIC (1:1.5) with above $10 \%$ power conversion efficiency. ${ }^{37-39}$ The solar cell parameters of these two blends are shown in Table 1. Moreover, to check the generality of our observations we investigated a large number of polymer and 

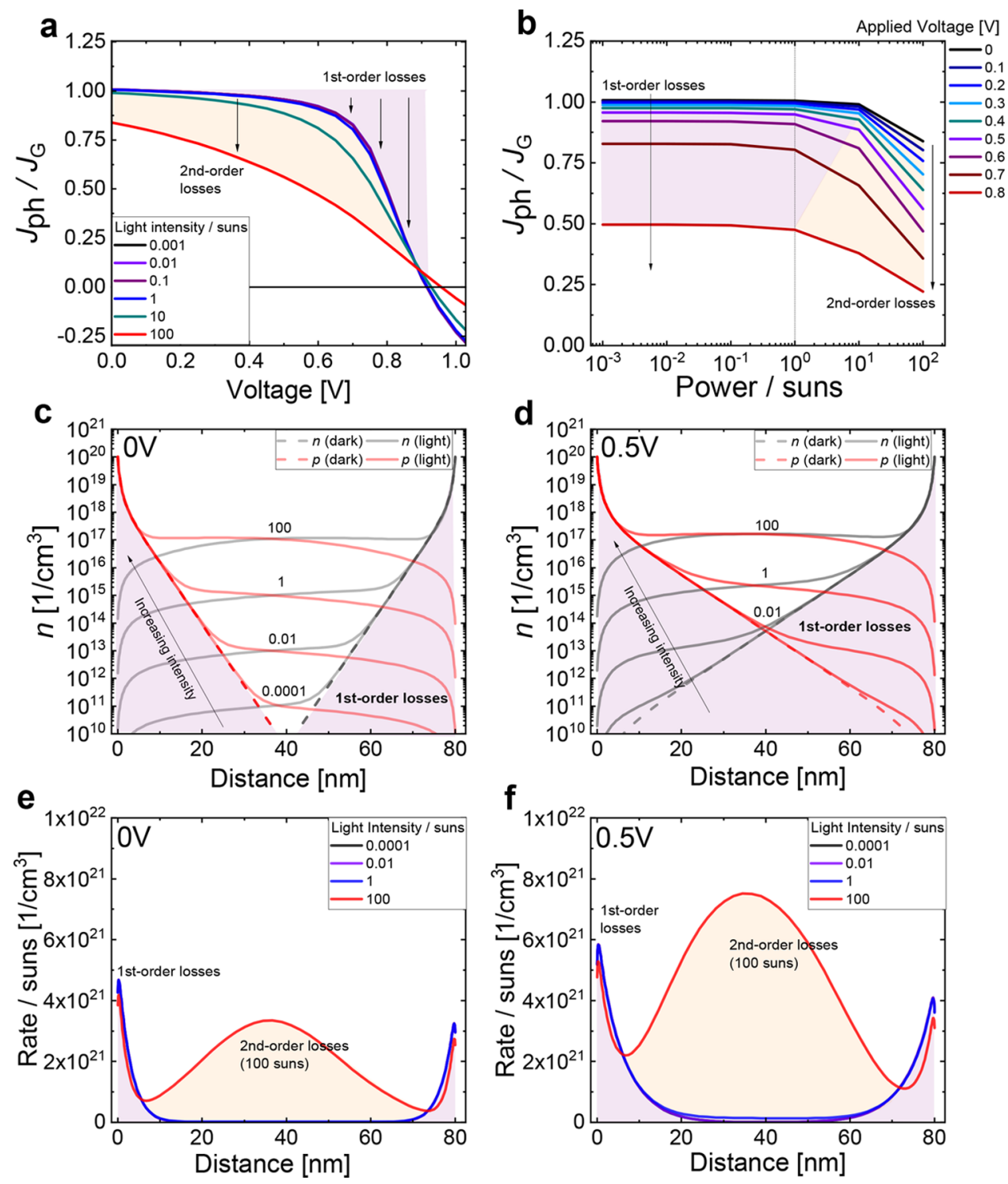

Figure 2. (a) Simulated photocurrent normalized to the generation current $\left(J_{\mathrm{ph}} / J_{\mathrm{G}}\right)$ vs $V$ curves at illumination intensities ranging from $10^{-3}$ to 100 suns. The decrease of FF above 1 sun marks the onset of second-order recombination losses, while the overlap of the $J_{\text {ph }} / J_{\mathrm{G}}$ vs $V$ curves at lower intensities shows the presence of first-order recombination losses. (b) Equivalent plot of $J_{\mathrm{ph}} / J_{\mathrm{G}}$ vs light intensity at different voltages. First-order recombination manifests as a decrease of the constant EQE with applied voltage independent of light intensity, while second-order recombination causes a downward bend of the EQE. The electron and hole densities are plotted at $0 \mathrm{~V}$ in panel (c) and at an applied bias of $0.5 \mathrm{~V}$ in panel (d). The populations of dark electrons and holes are then given by Fermi statistics depending on the energy difference between the conduction (valence) band and the Fermi level. The corresponding recombination profiles normalized by the generation rate along the vertical position in the active layer at $0 \mathrm{~V}(\mathrm{e})$ and $0.5 \mathrm{~V}(\mathrm{f})$ show first-order recombination near the contacts and second-order recombination emerging in the bulk at 100 suns.

small-molecule solar cells as shown in Figure S1. The chemical structures of all materials used in this study are shown in Figure S2.

In the PTB7:PC ${ }_{70} \mathrm{BM}$ blend, under short-circuit conditions, we observe a linear increase of the photocurrent with laser power (or equivalently a constant EQE) from $10^{-2}$ to approximately 5 suns (Figure 1a). This suggests the absence of higher-order recombination losses at short-circuit conditions and " 1 sun". However, in forward bias and at higher laser powers, nonlinear losses become apparent from the downward bend of the EQE. Under 1 sun conditions, it can be seen that the EQE losses originate almost exclusively from first-order recombination. Therefore, as shown in Figure $1 \mathrm{~b}$, the shape of the $J V$ curve in the power-generating regime is, in contrast to the common belief, largely controlled by linear recombination losses (in addition to the dark current). Figure 1c,d shows analogous measurements for a FTAZ:IDIC blend with close to $10 \%$ PCE. In order to check the influence of geminate recombination, i.e., field-dependent exciton or charge-transfer state dissociation, we conducted time delayed collection field (TDCF) experiments. ${ }^{4,9,34}$ For both cells, the external generation efficiency (EGE) is essentially flat as a function of the forward bias up to voltages close to $V_{\mathrm{OC}}$ as shown in Figure S3, which rules out a significant impact of geminate recombination on these first-order recombination losses. We also emphasize that the strong contribution of a recombination 

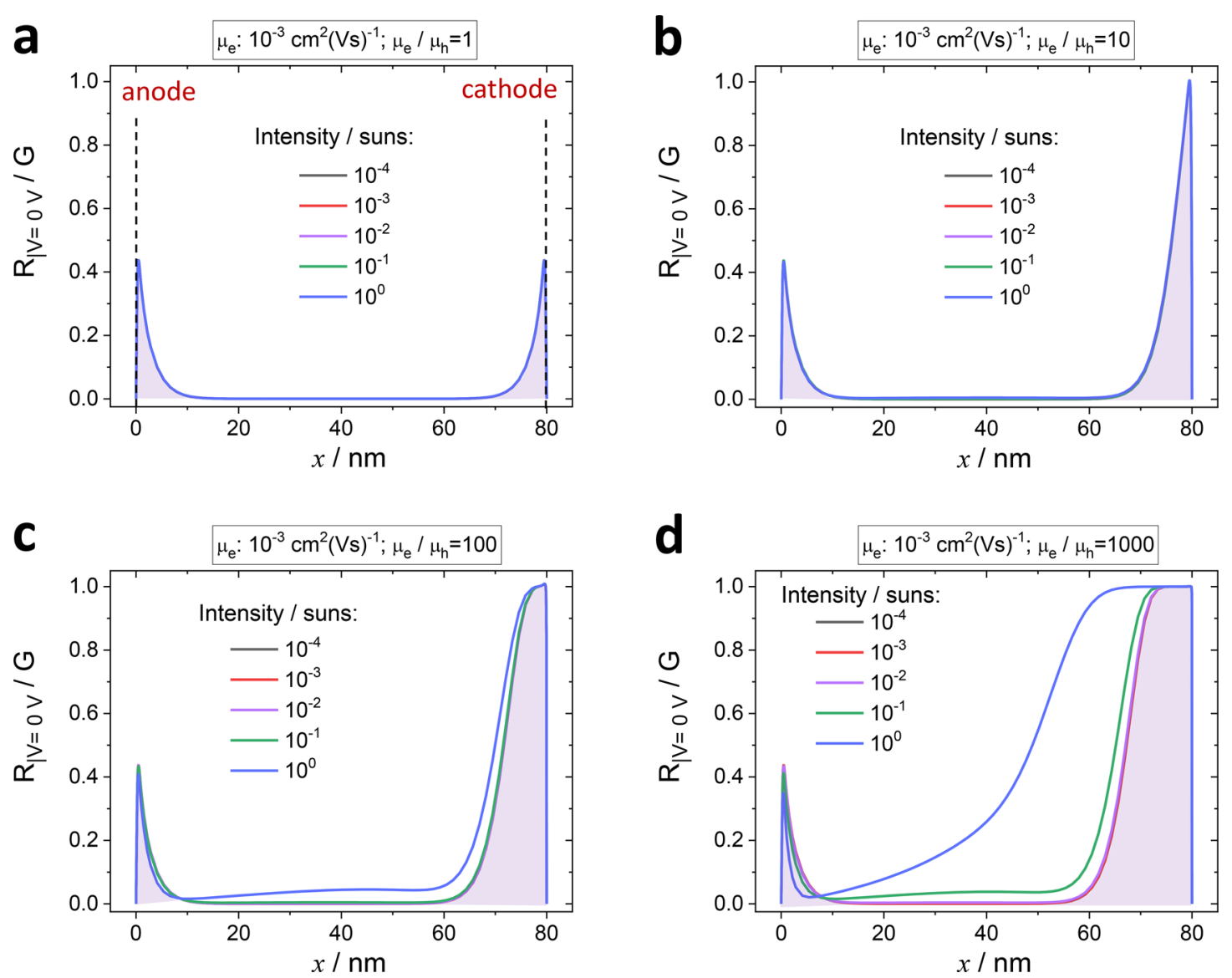

Figure 3. $(\mathrm{a}-\mathrm{d})$ Simulated recombination profiles at $0 \mathrm{~V}$ normalized by the generation rate along the active layer thickness for systems with decreasing slower carrier mobility $\left(\mu_{\mathrm{h}}=10^{-3}-10^{-6} \mathrm{~cm}^{2} \mathrm{~V}^{-1} \mathrm{~s}^{-1}\right)$ and constant electron mobility $\mu_{\mathrm{e}}=10^{-3} \mathrm{~cm}^{2} \mathrm{~V}^{-1} \mathrm{~s}^{-1}$. Losses that increase with intensity mark the onset of significant second-order recombination in panels $\mathrm{c}$ and $\mathrm{d}$, while the overlap of the curves at lower intensities shows the presence of first-order recombination losses (purple shading). The graph demonstrates that the linear and nonlinear recombination losses increase with decreasing mobility of the slower charge carriers.

loss which is strictly linear in light intensity appears in all optimized organic solar cells that we have studied so far, with several further examples shown in Figure S1.

Numerical Simulations: Origin of First-Order Recombination Losses. We first aimed to demonstrate the origin of these firstorder losses based on the example of the PTB7:PC ${ }_{70} \mathrm{BM}$ blend using drift-diffusion simulations based on bimolecular and surface recombination only. ${ }^{40,41}$ The used parameters are given in Table S1. For simplicity we assumed a homogeneous generation rate $G_{1 \mathrm{sun}}\left(\sim 1 \times 10^{22} \mathrm{~cm}^{-3} \mathrm{~s}^{-1}\right)$, no injection barriers for electrons and holes, and balanced mobilities $\mu_{\mathrm{e}, \mathrm{h}}=3 \times 10^{-4} \mathrm{~cm}^{2}(\mathrm{Vs})^{-1} \cdot{ }^{16,42}$ Figure 2a shows the photocurrent normalized to the generation current $\left(J_{\mathrm{ph}} / J_{\mathrm{G}}\right)$ as a function of voltage for four different illumination intensities, while Figure $2 \mathrm{~b}$ shows $J_{\mathrm{ph}} / J_{\mathrm{G}}$ as a function of illumination intensity for different applied voltages. The light intensityindependent $\mathrm{FF}$ of the $J_{\mathrm{ph}} / J_{\mathrm{G}}$ versus $V$ curves in Figure 2a indicates the presence of substantial first-order recombination (shaded purple area), while second-order recombination losses (shaded orange area) appear at higher intensities (>10 suns). Equivalently, linear and nonlinear losses are visible in the simulated IPC plot (Figure 2b). Thus, the simulations can accurately reproduce the presence of these recombination losses in the PTB7: $\mathrm{PC}_{70} \mathrm{BM}$ blend.
Figure $2 \mathrm{c}$,d reveals a high density of majority carriers near the electrodes in the bulk of the active layer even in the dark (shaded area), as discussed in several previous publications. ${ }^{43-46}$ These dark charges are simply a result of charge carrier diffusion from the metal electrodes into the (intrinsic) active layer, which leads to Fermi-level alignment and a built-in field across the active layer. This causes a light intensityindependent cloud of dark charges close to the contacts, which explains the first-order recombination rate close to the contacts. Notably, at intensities below 100 suns, these are also the regions with the strongest (normalized) recombination rate in the device, as shown in Figure $2 \mathrm{e}$ at $0 \mathrm{~V}$ and in Figure $2 \mathrm{f}$ at $0.5 \mathrm{~V}$. The overlap of the normalized recombination profiles reveals the presence of significant first-order recombination losses at all intensities $<1$ sun relatively close to the electrodes (approximately $15 \mathrm{~nm}$ at 0 $\mathrm{V})$. The recombination profiles also reveal that once a forward bias is applied, the carrier concentrations at the contacts ("+" at the anode, "-" at the cathode) are pushed deeper into the bulk of the active layer, which leads to an increased volume where bimolecular recombination of photogenerated with injected charges can take place (Figure 2f). This explains the reduction in normalized photocurrent in panels a and $b$. Importantly, this recombination mechanism is different from minority carrier surface recombination of photogenerated and dark charges at the electrodes which is, in this particular 

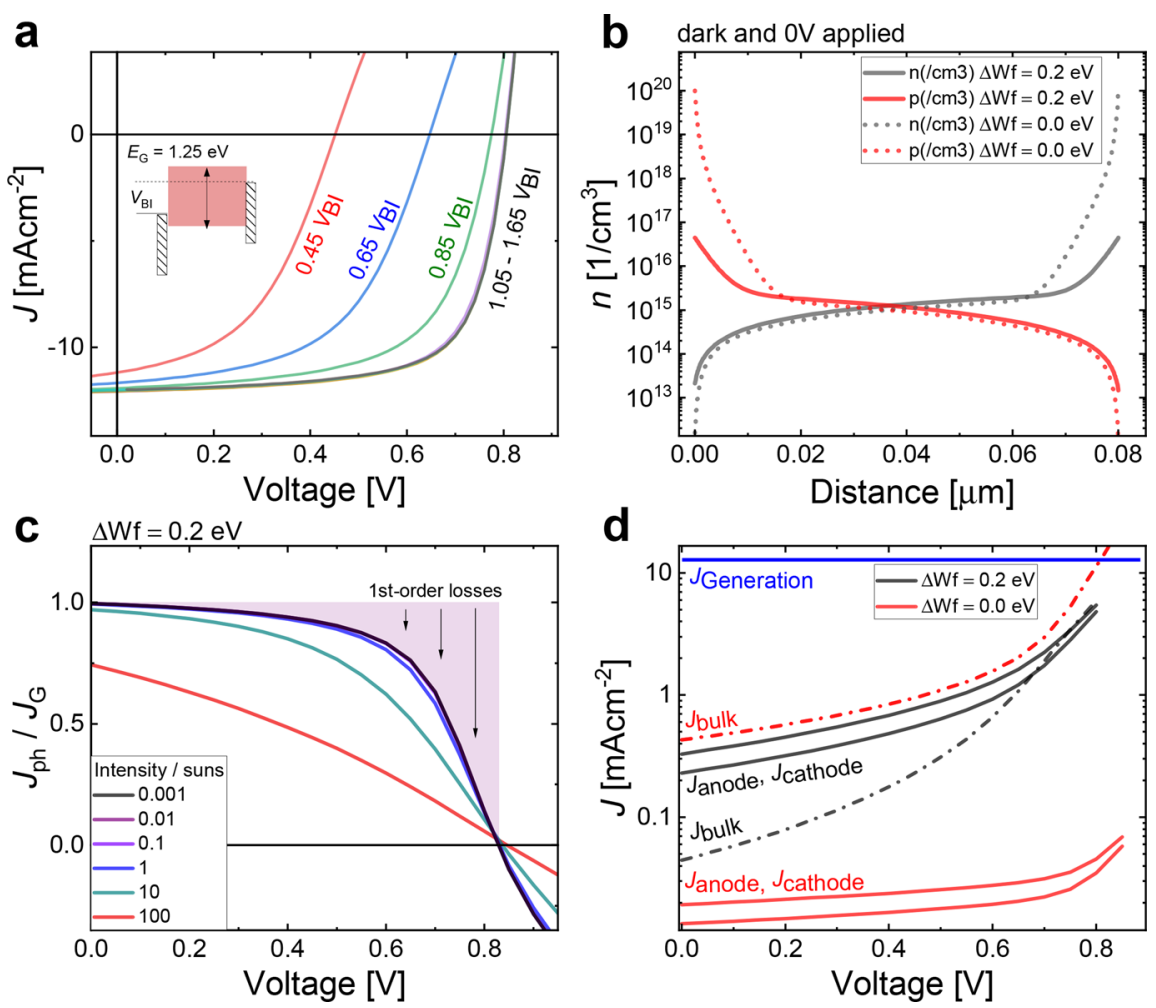

Figure 4. (a) Simulated $J V$ curves of organic solar cells with different energy offsets $\left(\Delta W_{\mathrm{f}}\right)$ between the metal work functions and the HOMO/ LUMO of the active layer. Ohmic contacts are beneficial for the $V_{\mathrm{OC}}$ and fill factor (FF) in the case of finite (and realistic) surface recombination velocities $(S>100 \mathrm{~cm} / \mathrm{s})$. (b) Impact of injection barriers on the carrier distribution profile in the dark. (c) Simulated $J_{\mathrm{ph}} / J_{\mathrm{G}}$ vs $V$ curves at illumination intensities ranging from $10^{-3}$ to 100 suns using $\Delta W_{\mathrm{f}}=0.2 \mathrm{eV}$ at both contacts. Even in the case of injection barriers, first-order losses dominate the $J V$ curve until 1 sun illumination because of enhanced surface recombination despite the reduction of first-order recombination in the bulk. This is shown in panel (d), which depicts the recombination currents at the metal surfaces and in the bulk as a function of voltage for two cases with and without injection barriers.

simulation, relatively small. This is further discussed below in Figure 4.

Impact of Carrier Mobility, Recombination Coefficient, and Film Thickness. It was previously observed in several polymer:fullerene cells that the magnitude of the first-order losses depends strongly on the charge carrier mobility of the slower carrier $\left(\mu_{\text {slower }}\right) .{ }^{16}$ To understand the detrimental impact of $\mu_{\text {slower, }}$ we varied the hole mobility over orders of magnitude $\left(10^{-3}-10^{-6} \mathrm{~cm}^{2} \mathrm{~V}^{-1} \mathrm{~s}^{-1}\right)$ while keeping the electron mobility constant at $\mu_{\mathrm{e}}=10^{-3} \mathrm{~cm}^{2} \mathrm{~V}^{-1} \mathrm{~s}^{-1}$. In Figure 3 we plot the normalized carrier recombination profile as a function of the vertical position $x$ in the device at $0 \mathrm{~V}$. We see that the recombination at the cathode increases with decreasing $\mu_{\mathrm{h}}$; that is, more photogenerated holes recombine with the large reservoir of injected electrons. It is also expected that the firstorder losses depend on the recombination rate constant $\left(k_{2}\right)$. Indeed, for a system with $100 \times$ imbalanced mobilities, a reduction of the Langevin recombination coefficient by 25 times greatly reduces the first-order losses, which in turn increases the FF of the cell (Figure S4). ${ }^{47,48}$ With respect to the impact of the active layer thickness, we observe that the first-order losses remain similar while second-order losses increase with the thickness of the active layer. This is expected considering that the volume in which first-order recombination takes place decreases relative to the volume of the bulk (Figure S5). This is also consistent with experimental IPC results on a nonoptimal FTAZ:IDIC (1:1.5) blend with an active layer thickness of $250 \mathrm{~nm}$ (Figure S6). For this device, we still observe large first-order recombination, however, with a significant contribution of second-order recombination throughout the power generating $J V$-regime. Therefore, these results suggest that first-order bimolecular recombination dominates the FF in OSCs with optimized film thicknesses, while in thicker blends, the contribution of additional secondorder recombination losses further deteriorates the FF.

Impact of the Electrode Work Functions. In the following section, we will discuss the impact of the contacts on these losses and potential optimization strategies by electrode engineering. ${ }^{44}$ First, we checked the influence of the energetic offset $\left(\Delta W_{\mathrm{f}}\right)$ between the metal work functions with respect to the highest (lowest) occupied (unoccupied) molecular orbitals (HOMO/LUMO) of the active layer material. Interestingly, although ohmic contacts $\left(\Delta W_{\mathrm{f}}=0 \mathrm{eV}\right)$ lead to more dark charge injection than injection barriers $\left(\Delta W_{\mathrm{f}}>0 \mathrm{eV}\right)$, energy level alignment between the electrodes and the active layer is actually beneficial to maximize the built-in voltage $\left(V_{\mathrm{BI}}\right)$ and in turn the cells' open-circuit voltage and FF. This is shown in Figure $4 \mathrm{a}$ from the $J V$ curves of cells where we varied $\Delta W_{\mathrm{f}}$ in steps of $100 \mathrm{mV}$ at both contacts. We note a sharp drop in $V_{\mathrm{OC}}$ if the $V_{\mathrm{BI}}$ drops below the $V_{\mathrm{OC}}$ that can be achieved by the blend $(\sim 0.8 \mathrm{~V})$ for the given device and material parameters such as $k_{2}, G$, and the surface recombination velocity $(S)$ which was set to $100 \mathrm{~cm} / \mathrm{s}$ for minority carriers (see Figure S7 for other settings). Figure $4 \mathrm{~b}$ demonstrates the large differences in the carrier densities close to the contacts with (solid lines) and 
without (dashed lines) injection barriers at both contacts ( 0.2 $\mathrm{eV})$. While we expected that these injection barriers will impact the interplay between linear and nonlinear recombination losses, surprisingly, also in the case with injection barriers of $0.2 \mathrm{eV}$ we still obtain significant first-order losses until 1 sun, which is shown in Figure 4c (note the similarities to the case of no injection barriers as shown in Figure $2 \mathrm{a}$ ). The reason is that surface recombination of minority carriers increases with the injection barrier offset, which is demonstrated in Figure 4d. We note that in the case with injection barriers $(0.2 \mathrm{eV})$, the optimized cell performance (with aligned energy levels) can be regained if the surface recombination velocity $S$ is reduced to very low values $<1 \mathrm{~cm} / \mathrm{s}$ (Figure $\mathrm{S} 7$ ). However, we can conclude that unless in special cases where $S$ is indeed close to 0 or where interlayers prevent surface recombination, ohmic contacts remain overall beneficial for the device performance because of the reduced surface recombination and the higher built-in field $\left(V_{\mathrm{BI}}\right)$ despite the strong first-order recombination in the vicinity of the electrodes. The impact of interlayers is further discussed in Figure S8. Lastly, it is important to note that although the first-order recombination in the bulk outweighs the surface recombination in the optimized case with $\Delta W_{\mathrm{f}}=0 \mathrm{eV}$, in general we do not know the relative contribution of these two loss mechanisms as it depends on the work function alignment and the relation between $S$ and $k_{2}$. However, both processes are similar in the sense that photogenerated charges recombine with electrode-induced charges near or at the metal contact, and as such we do not further attempt to disentangle these two first-order processes here.

In this work we studied the light intensity dependence of photocurrent and fill factor losses in OSCs using IPC measurements and numerical drift-diffusion simulations. Experimentally, we observed in all studied OSCs that the FF is almost entirely governed by first-order recombination losses, which means that the FF of the $J_{\mathrm{ph}}(V)$ curve does not improve significantly at intensities $<1$ sun. Our numerical simulations show consistently that these first-order losses can be very well reproduced by bimolecular recombination-without implementing trap-assisted Shockley-Read-Hall or geminate recombination. This is due to the large amount of majority charge carriers close to the metal contact, which is present independent of light intensity. With increasing forward bias, this excess charge is pushed deeper into the active layer of the material, causing increased first-order recombination losses which dominate the FF losses in optimized organic solar cells. We also showed that the magnitude of these linear recombination losses depends on the slower carrier mobility and the recombination coefficient, while enhancing the film thickness increases the contribution of second-order recombination losses in the bulk in unoptimized blends. Lastly, we discussed the impact of the electrode workfunctions on this recombination mechanism, which defines the relative loss due to surface recombination at the contacts and first-order recombination in the bulk. In this regard we identified that ohmic contacts are beneficial for the device performance as they maximize the $V_{\mathrm{BI}}$ despite causing more recombination between injected and photogenerated charges. Overall, our studies highlight the importance of bimolecular recombination between photogenerated and electrode-induced charges close to the metal contacts rather than bimolecular recombination of free charges in the whole bulk. This can explain the reason for the many different explanations that have been provided to explain FF losses in OSCs. The findings also have important consequences for the interpretation of several well-established characterization techniques: (1) A linear relationship between $J_{\mathrm{ph}}$ and $I$ (or the $\alpha$-parameter) cannot be used to draw conclusions about the dominance of monomolecular or bimolecular recombination at $0 \mathrm{~V}$. (2) Consequently, the deviation from linearity does not allow the quantification of the amount of bimolecular recombination losses (at $0 \mathrm{~V}$ ). (3) In forward bias, the linear relationship between $J_{\mathrm{ph}}$ and $I$ cannot be used to quantify the bias dependence of the charge generation yield or trap-assisted recombination. (4) FOMs that have been developed to characterize OSCs need to take into account the large impact of electrode-induced charges in the future.

\section{ASSOCIATED CONTENT}

\section{Supporting Information}

The Supporting Information is available free of charge on the ACS Publications website at DOI: 10.1021/acs.jpclett.9b01175.

Details of the experimental and numerical methods, a note about the terminology with respect to the recombination type and order, IPC measurements on other organic solar cell systems, chemical structures of the materials studied in this work, TDCF measurements, table with the parameters used for the numerical drift diffusion simulations, further simulation results on the impact of the recombination coefficient and film thickness, IPC measurements on thicker FTAZ:IDIC blends, and numerical simulations regarding the impact of the surface recombination velocity and interlayers (PDF)

\section{AUTHOR INFORMATION}

\section{Corresponding Authors}

*E-mail: uli.wuerfel@ise.fraunhofer.de.

*E-mail: stolterf@uni-potsdam.de.

ORCID

Lorena Perdigón-Toro: 0000-0002-0957-2027

Christian M. Wolff: 0000-0002-7210-1869

Jeromy James Rech: 0000-0001-7963-9357

Xiaowei Zhan: 0000-0002-1006-3342

Wei You: 0000-0003-0354-1948

Safa Shoaee: 0000-0001-5754-834X

Dieter Neher: 0000-0001-6618-8403

Martin Stolterfoht: 0000-0002-4023-2178

\section{Notes}

The authors declare no competing financial interest.

\section{ACKNOWLEDGMENTS}

J.J.R. is supported by NSF grant (CBET-1639429). S.S. is supported by a Sofia Kovalevskaya Award from the Alexander von Humboldt Foundation. P.C. is funded by HyPerCells - a joint graduate school of the Potsdam University and the HZB. Further support came from the Deutsche Forschungsgesellschaft (DFG) via NE 410/13-1, NE410/15-1, and INST 336/ 94-1 FUGG. We thank Fuwen Zhao from the Peking University for providing details regarding the fabrication of FTAZ:IDIC devices. 


\section{REFERENCES}

(1) Zhou, H.; Zhang, Y.; Seifter, J.; Collins, S. D.; Luo, C.; Bazan, G. C.; Nguyen, T.-Q.; Heeger, A. J. High-Efficiency Polymer Solar Cells Enhanced by Solvent Treatment. Adv. Mater. 2013, 25 (11), 16461652.

(2) Lu, L.; Zheng, T.; Wu, Q.; Schneider, A. M.; Zhao, D.; Yu, L. Recent Advances in Bulk Heterojunction Polymer Solar Cells. Chem. Rev. 2015, 115 (23), 12666-12731.

(3) Hou, J.; Inganas, O.; Friend, R. H.; Gao, F. Organic Solar Cells Based on Non-Fullerene Acceptors. Nat. Mater. 2018, 17 (2), 119128.

(4) Firdaus, Y.; Le Corre, V. M.; Khan, J. I.; Kan, Z.; Laquai, F.; Beaujuge, P. M.; Anthopoulos, T. D. Key Parameters Requirements for Non-Fullerene-Based Organic Solar Cells with Power Conversion Efficiency > 20\%. Adv. Sci. 2019, 6, 1802028.

(5) Yuan, J.; Zhang, Y.; Zhou, L.; Zhang, G.; Yip, H.-L.; Lau, T.-K.; Lu, X.; Zhu, C.; Peng, H.; Johnson, P. A.; et al. Single-Junction Organic Solar Cell with over 15\% Efficiency Using Fused-Ring Acceptor with Electron-Deficient Core. Joule 2019, 3, 1140.

(6) Würfel, U.; Neher, D.; Spies, A.; Albrecht, S. Impact of Charge Transport on Current-Voltage Characteristics and Power-Conversion Efficiency of Organic Solar Cells. Nat. Commun. 2015, 6 (6951), 6951.

(7) Neher, D.; Kniepert, J.; Elimelech, A.; Koster, L. J. A. A New Figure of Merit for Organic Solar Cells with Transport-Limited Photocurrents. Sci. Rep. 2016, 6, 24861.

(8) Lakhwani, G.; Rao, A.; Friend, R. H. Bimolecular Recombination in Organic Photovoltaics. Annu. Rev. Phys. Chem. 2014, 65 (1), 557581.

(9) Bartesaghi, D.; Pérez, I. D. C.; Kniepert, J.; Roland, S.; Turbiez, M.; Neher, D.; Koster, L. J. A. Competition between Recombination and Extraction of Free Charges Determines the Fill Factor of Organic Solar Cells. Nat. Commun. 2015, 6 (1), 7083.

(10) Kirchartz, T.; Agostinelli, T.; Campoy-Quiles, M.; Gong, W.; Nelson, J. Understanding the Thickness-Dependent Performance of Organic Bulk Heterojunction Solar Cells: The Influence of Mobility, Lifetime, and Space Charge. J. Phys. Chem. Lett. 2012, 3 (23), 34703475.

(11) Stolterfoht, M.; Armin, A.; Philippa, B.; Neher, D. The Role of Space Charge Effects on the Competition between Recombination and Extraction in Solar Cells with Low-Mobility Photoactive Layers. J. Phys. Chem. Lett. 2016, 7 (22), 4716-4721.

(12) Koster, L. J. A.; Kemerink, M.; Wienk, M. M.; Maturová, K.; Janssen, R. A. J. Quantifying Bimolecular Recombination Losses in Organic Bulk Heterojunction Solar Cells. Adv. Mater. 2011, 23 (14), $1670-1674$.

(13) Cowan, S. R.; Roy, A.; Heeger, A. J. Recombination in PolymerFullerene Bulk Heterojunction Solar Cells. Phys. Rev. B: Condens. Matter Mater. Phys. 2010, 82 (24), 245207.

(14) Li, Z.; McNeill, C. R. Transient Photocurrent Measurements of PCDTBT:PC70BM and PCPDTBT:PC70BM Solar Cells: Evidence for Charge Trapping in Efficient Polymer/Fullerene Blends. J. Appl. Phys. 2011, 109 (7), 074513.

(15) Street, R. a.; Schoendorf, M.; Roy, A.; Lee, J. H. Interface State Recombination in Organic Solar Cells. Phys. Rev. B: Condens. Matter Mater. Phys. 2010, 81 (20), 205307.

(16) Stolterfoht, M.; Shoaee, S.; Armin, A.; Jin, H.; Kassal, I.; Jiang, W.; Burn, P.; Meredith, P. Electric Field and Mobility Dependent First-Order Recombination Losses in Organic Solar Cells. Adv. Energy Mater. 2017, 7 (4), 1601379.

(17) Bin, H.; Gao, L.; Zhang, Z. G.; Yang, Y.; Zhang, Y.; Zhang, C.; Chen, S.; Xue, L.; Yang, C.; Xiao, M.; et al. 11.4\% Efficiency NonFullerene Polymer Solar Cells with Trialkylsilyl Substituted 2DConjugated Polymer as Donor. Nat. Commun. 2016, 7 (1), 13651.

(18) Yuan, J.; Zhang, Y.; Xie, S.; Zhang, X.; Zuo, X.; Zhou, H. Understanding Charge Transport and Recombination Losses in High Performance Polymer Solar Cells with Non-Fullerene Acceptors. J. Mater. Chem. A 2017, 5 (33), 17230-17239.
(19) Gupta, V.; Leong, W. L.; Bazan, G. C.; Kyaw, A. K. K.; Wang, D. H.; Heeger, A. J.; Ke, L. Intensity Dependence of Current-Voltage Characteristics and Recombination in High-Efficiency SolutionProcessed Small-Molecule Solar Cells. ACS Nano 2013, 7 (5), 4569-4577.

(20) Cowan, S. R.; Wang, J.; Yi, J.; Lee, Y. J.; Olson, D. C.; Hsu, J. W. P. Intensity and Wavelength Dependence of Bimolecular Recombination in P3HT:PCBM Solar Cells: A White-Light Biased External Quantum Efficiency Study. J. Appl. Phys. 2013, 113 (15), 154504.

(21) Stolterfoht, M.; Armin, A.; Philippa, B.; White, R. D.; Burn, P. L.; Meredith, P.; Juška, G.; Pivrikas, A.; et al. Photocarrier Drift Distance in Organic Solar Cells and Photodetectors. Sci. Rep. 2015, 5 (1), 9949.

(22) Cowan, S. R.; Leong, W. L.; Banerji, N.; Dennler, G.; Heeger, A. J. Identifying a Threshold Impurity Level for Organic Solar Cells: Enhanced First-Order Recombination Via Well-Defined PC84BM Traps in Organic Bulk Heterojunction Solar Cells. Adv. Funct. Mater. 2011, 21 (16), 3083-3092.

(23) Li, Z.; Lakhwani, G.; Greenham, N. C.; McNeill, C. R. VoltageDependent Photocurrent Transients of PTB7:PC70BM Solar Cells: Experiment and Numerical Simulation. J. Appl. Phys. 2013, 114 (3), 034502 .

(24) Kyaw, A. K. K.; Wang, D. H.; Gupta, V.; Leong, W. L.; Ke, L.; Bazan, G. C.; Heeger, A. J. Intensity Dependence of Current À Voltage Characteristics and Recombination in. ACS Nano 2013, 7 (5), 4569-4577.

(25) Dibb, G. F. A.; Kirchartz, T.; Credgington, D.; Durrant, J. R.; Nelson, J. Analysis of the Relationship between Linearity of Corrected Photocurrent and the Order of Recombination in Organic Solar Cells. J. Phys. Chem. Lett. 2011, 2 (19), 2407-2411.

(26) Mihailetchi, V. D.; Koster, L. J. A.; Hummelen, J. C.; Blom, P. W. M. Photocurrent Generation in Polymer-Fullerene Bulk Heterojunctions. Phys. Rev. Lett. 2004, 93 (21), 216601.

(27) Lenes, M.; Morana, M.; Brabec, C. J.; Blom, P. W. M. Recombination-Limited Photocurrents in Low Bandgap Polymer/ Fullerene Solar Cells. Adv. Funct. Mater. 2009, 19 (7), 1106-1111.

(28) Hahn, T.; Tscheuschner, S.; Kahle, F. J.; Reichenberger, M.; Athanasopoulos, S.; Saller, C.; Bazan, G. C.; Nguyen, T. Q.; Strohriegl, P.; Bässler, H. Monomolecular and Bimolecular Recombination of Electron-Hole Pairs at the Interface of a Bilayer Organic Solar Cell. Adv. Funct. Mater. 2017, 27 (1), 1604906.

(29) Kniepert, J.; Schubert, M.; Blakesley, J. C.; Neher, D. Photogeneration and Recombination in P3HT/PCBM Solar Cells Probed by Time-Delayed Collection Field Experiments. J. Phys. Chem. Lett. 2011, 2 (7), 700-705.

(30) Mauer, R.; Howard, I. A.; Laquai, F. F. Effect of External Bias on Nongeminate Recombination in Polythiophene/Methanofullerene Organic Solar Cells. J. Phys. Chem. Lett. 2011, 2 (14), 1736-1741.

(31) Foertig, A.; Kniepert, J.; Gluecker, M.; Brenner, T.; Dyakonov, V.; Neher, D.; Deibel, C. Nongeminate and Geminate Recombination in PTB7:PCBM Solar Cells. Adv. Funct. Mater. 2014, 24, 1306.

(32) Gélinas, S.; Rao, A.; Kumar, A.; Smith, S. L.; Chin, A. W.; Clark, J.; van der Poll, T. S.; Bazan, G. C.; Friend, R. H. Ultrafast Long-Range Charge Separation in Organic Semiconductor Photovoltaic Diodes. Science (Washington, DC, U. S.) 2014, 343 (6170), $512-516$.

(33) Dibb, G. F. A.; Jamieson, F. C.; Maurano, A.; Nelson, J.; Durrant, J. R. Limits on the Fill Factor in Organic Photovoltaics: Distinguishing Nongeminate and Geminate Recombination Mechanisms. J. Phys. Chem. Lett. 2013, 4 (5), 803-808.

(34) Vandewal, K.; Albrecht, S.; Hoke, E. T.; Graham, K. R.; Widmer, J.; Douglas, J. D.; Schubert, M.; Mateker, W. R.; Bloking, J. T.; Burkhard, G. F.; et al. Efficient Charge Generation by Relaxed Charge-Transfer States at Organic Interfaces. Nat. Mater. 2014, 13 (1), 63-68.

(35) Stolterfoht, M.; Armin, A.; Shoaee, S.; Kassal, I.; Burn, P.; Meredith, P. Slower Carriers Limit Charge Generation in Organic 
Semiconductor Light-Harvesting Systems. Nat. Commun. 2016, 7 (1), 11944.

(36) Liang, Y.; Xu, Z.; Xia, J.; Tsai, S.-T.; Wu, Y.; Li, G.; Ray, C.; Yu, L. For the Bright Future - Bulk Heterojunction Polymer Solar Cells with Power Conversion Efficiency of 7.4\%. Adv. Mater. 2010, 22 (20), E135-E138.

(37) Lin, Y.; Zhao, F.; Prasad, S. K. K.; Chen, J.-D.; Cai, W.; Zhang, Q.; Chen, K.; Wu, Y.; Ma, W.; Gao, F.; et al. Balanced Partnership between Donor and Acceptor Components in Nonfullerene Organic Solar Cells with > 12\% Efficiency. Adv. Mater. 2018, 30 (16), 1706363.

(38) Price, S. C.; Stuart, A. C.; Yang, L.; Zhou, H.; You, W. Fluorine Substituted Conjugated Polymer of Medium Band Gap Yields 7\% Efficiency in Polymer-Fullerene Solar Cells. J. Am. Chem. Soc. 2011, 133 (12), 4625-4631.

(39) Zhao, W.; Li, S.; Yao, H.; Zhang, S.; Zhang, Y.; Yang, B.; Hou, J. Molecular Optimization Enables over 13\% Efficiency in Organic Solar Cells. J. Am. Chem. Soc. 2017, 139 (21), 7148-7151.

(40) Synopsys. Synopsys TCAD, release L-2016.03-SP22016. Available: https://www.synopsys.com/silicon/tcad.html.

(41) Burgelman, M.; Nollet, P.; Degrave, S. Modelling Polycrystalline Semiconductor Solar Cells. Thin Solid Films 2000, 361, 527-532.

(42) Philippa, B.; Stolterfoht, M.; Burn, P. L.; Juška, G.; Meredith, P.; White, R. D.; Pivrikas, A. The Impact of Hot Charge Carrier Mobility on Photocurrent Losses in Polymer-Based Solar Cells. Sci. Rep. 2015, 4 (1), 5695.

(43) Rahimi Chatri, A.; Torabi, S.; Le Corre, V. M.; Koster, L. J. A. Impact of Electrodes on Recombination in Bulk Heterojunction Organic Solar Cells. ACS Appl. Mater. Interfaces 2018, 10 (14), 12013-12020.

(44) Reinhardt, J.; Grein, M.; Bühler, C.; Schubert, M.; Würfel, U. Identifying the Impact of Surface Recombination at Electrodes in Organic Solar Cells by Means of Electroluminescence and Modeling. Adv. Energy Mater. 2014, 4 (11), 1400081.

(45) Wehenkel, D. J.; Koster, L. J. A.; Wienk, M. M.; Janssen, R. A. J. Influence of Injected Charge Carriers on Photocurrents in Polymer Solar Cells. Phys. Rev. B: Condens. Matter Mater. Phys. 2012, 85 (12), $1-12$.

(46) Wetzelaer, G. A. H.; Blom, P. W. M. Ohmic Current in Organic Metal-Insulator-Metal Diodes Revisited. Phys. Rev. B: Condens. Matter Mater. Phys. 2014, 89 (24), 241201.

(47) Heiber, M. C.; Baumbach, C.; Dyakonov, V.; Deibel, C. Encounter-Limited Charge-Carrier Recombination in Phase-Separated Organic Semiconductor Blends. Phys. Rev. Lett. 2015, 114 (13), 136602.

(48) Burke, T. M.; Sweetnam, S.; Vandewal, K.; McGehee, M. D. Beyond Langevin Recombination: How Equilibrium Between Free Carriers and Charge Transfer States Determines the Open-Circuit Voltage of Organic Solar Cells. Adv. Energy Mater. 2015, 5 (11), 1500123. 